\title{
The nature and mathematical basis for material stability in the chemical and biological worlds
}

\author{
Robert Pascal ${ }^{1}$ and Addy Pross ${ }^{2^{*}}$
}

\begin{abstract}
The conceptual divide separating the physical and biological sciences continues to challenge modern science. In this perspective it is proposed that the two sciences can be directly connected through the fundamental concept of stability. Physicochemical stability is shown to have a logical, rather than an empirical basis, and able to manifest itself in two distinct and often contrary ways, one thermodynamic, reflecting energetic considerations, and the other kinetic, reflecting time/persistence considerations. Each stability kind is shown to rest on a particular mathematical truism. Thermodynamic stability, the energetic expression, has a probabilistic/statistical basis due to Boltzmann, and leads to the Second Law of Thermodynamics. Dynamic kinetic stability (DKS), the time/persistence expression, is attributed to the stability associated with persistent replicating systems, and derives from the mathematics of exponential growth. The existence of two distinct stability kinds, each mathematically-based, leads to two distinct organizational forms of matter, animate and inanimate. That understanding offers insight into the reasons for the observation of just those two organizational forms, their different material characteristics, and provides a logical basis for understanding the nature of chemical and biological transformations, both within, and between, the two forms.
\end{abstract}

Keywords: Thermodynamic stability, Dynamic kinetic stability, Origin of life, Autocatalysis, Abiogenesis

\section{Report Introduction}

The question of why matter exists in two starkly distinct material categories - living and non-living - has puzzled mankind for millennia. Our understanding of the living world was, of course, revolutionized through Darwin's landmark ideas of natural selection and common descent [1], and that understanding has been both deepened and extended by the dramatic advances in molecular biology over the past 60 years. Yet, despite those insights into the workings of life's molecular machinery, the perennial and more general question - how living and nonliving relate to one another - continues to elude us. Why does matter exist in two distinctly different organizational forms? Why not just one (or three)? Is there some basis in the laws of nature which would make the existence of two distinct forms expected, even inevitable? And with regard to those living forms, what would the

\footnotetext{
* Correspondence: pross@bgu.ac.il

${ }^{2}$ Department of Chemistry, Ben-Gurion University of the Negev, Be'er Sheva 84105, Israel

Full list of author information is available at the end of the article
}

material prerequisites for a generalized living system be? What is it about living systems that makes their properties so different to those of non-living ones? And, finally, of the two organizational forms, which is naturally preferred, and why? Such questions are not merely theoretical. Being able to understand the relationship between those two material forms would be a prerequisite for understanding how, at least in principle, one would go about transforming one form to the other. Needless to say, it is trivially easy to convert animate to inanimate, yet how tantalizingly difficult to proceed in the opposite direction. And the problem does not lie with a particular technical difficulty in one particular step along the way. The problem is much deeper. Despite those 60 years of mechanistic advances in molecular biology, the essence of the living state continues to elude us. As Kauffman [2] put it recently: "...we know many of the parts and many of the processes. But what makes a cell alive is still not clear to us. The center is still mysterious".

Then there is the perennial origin of life problem, fascinating in its own right [3-10]. How and why did this distinct organizational form of matter emerge in the first
() Chemistry Central 
place? There is a broad scientific consensus that life on earth emerged from inanimate beginnings, and at first sight the origin of life question might appear unrelated to the other life questions, but that impression is false. While the historic path linking inanimate to animate will likely remain buried in the mists of time $[4,5]$, the question how life could have emerged from inanimate matter is intimately connected to the means by which one would go about synthesizing a living system. The two questions constitute two sides of the one coin; solve one and you've taken a major step toward solving the other. By what process and based on what physicochemical principles was it possible for matter to be transformed from the relatively well-understood inanimate state into that extraordinarily complex and thermodynamically unstable animate state. Certainly from a purely thermodynamic perspective such a transition would seem to be spectacularly improbable [11-13].

But the confusion surrounding the living state goes further. Consider the extraordinary characteristics of living systems, which seem to defy simple chemical explanation. Whereas chemistry is readily able to explain the characteristics of inanimate materials - why ice is hard, why metals conduct electricity, why helium is chemically inert and a gas at room temperature, and so on, living matter's strikingly different properties do not lend themselves to that kind of chemical approach [5]. Take the concept of function, for example, one that permeates all of biology. There is an entire area of biology, functional biology, which continually asks 'what is its function, how does it work' type questions, as the purposeful (teleonomic) character of living systems is empirically irrefutable. But how is it at all possible for any biological system, ultimately just chemical in its composition, able to express the characteristic we denote by the term function, which of necessity also signifies purpose? In the inanimate world, in the world of 'regular' chemistry there is no function, no purpose. That, after all, was the essence of the scientific revolution of the 17th century. Teleology was banished from the scientific discourse [14]. How then are biological systems, entirely material in nature, able to manifest function? In the context of the origin of life question, the issue can be rephrased as: how could biological function have emerged from an inanimate world devoid of function? How could biological systems have acquired properties seemingly inconsistent with nature's objective character?

And, finally, to the heart of the problem - the nature of biological organization, as it is within that special kind of organization that the essence of the animate - inanimate distinction presumably needs to be sought. How can biological organization as a phenomenon, characterized by inordinate dynamic (homeostatic) complexity and quite distinct to the static complexity of the inanimate world, be understood, an issue glossed over in the neoDarwinian view? In response to these probing questions, directed toward clarifying the nature of the chemistrybiology connection, modern biology has taken a defensive posture and battened down the hatches. The unstated but implicit message in contemporary biology appears to be: yes, there are innumerable apparent contradictions when biology is directly confronted with physics and chemistry [2,11-14]. However, since the physical sciences have not provided biology with the appropriate conceptual and methodological tools for resolving these contradictions, biology can avoid these awkward questions by fencing itself off from the physical sciences. The result: biology of the 20th century has been overtaken by an 'autonomy of biology' philosophy, one openly endorsed by Ernst Mayr [15], one of the leading evolutionary biologists of the 20th century, whereby biology is treated as a disparate science governed by a separate philosophy to the one underpinning the physical sciences. There are two kinds of matter, inanimate and animate, the physical sciences deal with the former, the biological sciences deal with the latter, and that's that! Thus in the neo-Darwinian perspective, biology's essence resides in the genome and the information coded therein, and from this vantage point, questions of origins - how did genomic information come about, how does information emerge from non-information - are conveniently brushed aside. But if, as is now widely believed, on planet Earth some 3.5 to 4 billion years ago chemistry did become biology [3-10], then the two subjects must in some sense be one, making it clear that the historical merging that took place in the distant past must be accompanied by a corresponding conceptual merging. The dissonance that continues to radiate from the glaring contradictions inherent in the biological and physical world views gives no respite.

In several recent papers the authors, together and separately, have attempted to address these questions, to help bridge the chemistry-biology gap, through the characterization of a unique stability kind in nature, termed dynamic kinetic stability (DKS) [4,5,12,13,16-19]. This stability kind, quite distinct to traditional thermodynamic stability, applies to systems able to maintain a presence over time through a process of self-replication. Thus replicative stability, whether chemical or biological, is able to lead to a distinct and separate organizational state - a kinetic state of matter, thereby offering a physicochemical framework for relating biological systems to replicative chemical ones. Through that approach several of the puzzling issues regarding the relationship of animate to inanimate appear resolvable - the continuity and underlying unity of chemical and biological evolution $[4,5,13,16,17]$, its physicochemical characterization $[12,13]$, the source of life's functional nature [20], its extraordinary and distinct kind of complexity [20], its 
metabolic (energy-consuming) character [12,13,21], to mention central ones.

In this paper we wish to refine and extend the argument regarding the nature of stability in the physicochemical world by pointing out that the concept of stability can be logically defined, and that the two stability kinds that govern physicochemical processes in the inanimate and animate worlds - thermodynamic stability and DKS respectively, are not arbitrary and empirically derived, but have a mathematical basis. Through an understanding of that basis for the two respective material forms, insight is offered into why there are two organizational material forms in nature, why the animate state once formed is inherently preferred over the inanimate state, and a clearer understanding as to why the origin of life question (meaning that initial transformation of inanimate to animate) is continuing to prove so intractable.

\section{Discussion}

\section{The nature of stability}

Let us begin by considering what the term 'stability' actually means within a physicochemical context. Our starting point is the observation that matter is not immutable, that the material world is undergoing continual change. That statement is, of course, empirically selfevident. Wherever one looks in the world, one can discern change, both physical and chemical. Significantly, however the direction of change can be summed up by the qualitative statement: all physicochemical systems tend from less stable to more stable forms. This general statement, not normally discussed (though alluded to by Dawkins [22]) may be thought of as axiomatic. It is inherent in the definition of the term 'stable' - unchanging, persistent over time. The statement is axiomatic in the sense that it is tautological to state that changing systems change, whereas unchanging ones do not. But within that tautology lies hidden a deeper truth. It is implicit that if matter does tend to undergo change, over time that change will necessarily be in the direction from systems more susceptible to change (i.e., less stable/persistent forms), toward systems that are less susceptible to change (i.e., more stable/persistent forms). Indeed, even if at some point the system were to change in the reverse direction, namely, from a relatively unstable form to a form that is even less stable, then, by definition, that change would be transitory, as the system would change yet again (by definition), until reaching a more stable form, one less susceptible to change (in the present context change is understood as one that is spontaneous, without the work or action of an external agent). Thus the direction of change is implicit in the very definition of stability. Stability is logically rather than empirically defined.
Note, however, that the above discussion has to an extent switched the concept of stability, normally associated with a system's energy to one that focuses on the system's persistence, i.e., its stability over time. The question then arises: is the stability of a system manifest through its energetic properties, or by its unchanging character over time, regardless of energetic considerations? As we will now discuss, stability in its energetic sense necessarily leads to stability in its time (persistence) sense, but not all systems that are stable in a time (persistent) sense, are necessarily stable in an energetic sense.

\section{Existence of two stability kinds}

The concept of stability as part of our consideration of the physicochemical world is of course fundamental and well-established, but the focus tends to be on just one kind of stability - thermodynamic stability, a stability kind associated with a system's energy Accordingly, the general 'less stable to more stable' rule described earlier is expressed by the Second Law of Thermodynamics, a law which formalizes the stability concept by providing a means for its quantification. And being the rule that specifies the direction of all irreversible processes, there is no doubting the Second Law's status as one of the fundamental tenets of physics and chemistry, one that operates at both macroscopic and cosmological levels. Of course the Second Law is not merely an empirical law, even though it was initially formulated as such by Clausius and Kelvin, but, as Boltzmann pointed out over a century ago, there is a mathematical logic, a mathematical underpinning to the law with the concept of entropy as its centerpiece [23]. Thus the most stable macrostate of a system (in energy terms) is the one described by the largest number of contributing microstates and the Second Law formulation of 'less stable to more stable' can be restated more insightfully as 'less probable to more probable'. Indeed, it is that inherent mathematical/statistical logic that elevates the broader concept of stability from one that is merely qualitative to one that is quantitative, thereby giving the law its almost hallowed status as one that is supremely incontestable. Importantly, a system that is stable in this energetic sense will also be stable in a time (persistence) sense. A system that has reached its lowest energy state, the equilibrium state, remains unchanged over time; energetic stability invariably leads to time stability (persistence).

Though energetic stability necessarily leads to time stability, the reverse does not necessarily apply. A system may well be stable in a time sense (persistent) without being stable in an energy sense. The familiar concept of kinetic stability characterizes that other stability kind, as exemplified by a hydrogen and oxygen gas mixture. Such a mixture is highly unstable in an energetic sense (a 
spark or catalyst will immediately result in water formation), but can be highly stable in a time sense - a mixture of the two gases may well persist over long periods of time.

But, as noted earlier, within the biological world as well as parts of the chemical world, an alternative kinetic stability kind exists and governs the nature of transformations within that world - DKS, a stability kind associated solely with the replicative world, and distinct to the more familiar static kinetic stability mentioned above. Indeed it is that concept of DKS that can help explain both replicative chemical, as well as biological, phenomena. Accordingly, replicating systems, though unstable in thermodynamic terms, are able to persist over time through continuing self-replication, and so are stable in kinetic terms. They are stable, not because they do not react, but because they $d o$ - to make more of themselves - thereby opening a door to a distinctly different organizational form of matter $[4,5,13,16,18]$.

But what is the basis for this other stability kind? Is it just empirical, or is there some underlying imperative that enables it to circumvent the probabilistic drive of the Second Law? Does the DKS concept also express some underlying, but alternative mathematical logic, to the probabilistic one? The answer to this last question appears to be yes, DKS is also governed by a mathematicallybased directive - the enormous kinetic power associated with systems able to undergo exponential growth due to the kinetic character of some (though not all) autocatalytic systems [24,25]. The central role of autocatalysis in the emergence of life has long been recognized and has been described within different theoretical models [26-28]. And, indeed, it is the kinetic power associated with autocatalysis which initiates the beginning of divergence from a thermodynamically-directed world by the establishment of what is effectively a parallel kinetic world in which systems are found to be dynamic, energy consuming, farfrom-equilibrium, and necessarily open to material and energy resources $[12,13]$. Let us describe how this comes about.

Once a DKS state does emerge, it turns out that its key reactivity characteristic, its potential ability to evolve, is also governed by that same mathematical directive. Due to the action of the Second Law, a stable DKS system will over time necessarily undergo variation leading to competition between the variants for resources. It has been recognized since Lotka [29] that autocatalytic systems can exhibit a range of complex kinetic behaviors [30-32], but it was Lifson [33] who explicitly pointed out that two competing autocatalysts that exhibit exponential growth and feed off common resources cannot coexist. Solution of the relevant rate equations leads to an unambiguous result - the more stable replicator (in the time/ persistent sense) drives the less stable one into extinction.
Of course, for the above evolutionary mechanism to be operative, the DKS system must be inherently evolvable $[34,35]$. Thus an autocatalytic network of reactions, such as the one involved in the formose reaction [36], would not satisfy this condition, as it lacks any possibility of evolving toward a state of increased DKS. Similarly, a system involving the autocatalytic production of fatty acids leading to vesicle division [37] would also be unable to satisfy this condition. But once evolvability is present within the system, such as is naturally found in template-based biopolymeric replicating systems, the DKS formulation opens up a mechanism for the stabilization of inherently less stable replicating entities. In fact the drive toward greater DKS can be expected to favor those systems whose evolvability is greater, so that initially weak evolvability will itself likely evolve into stronger evolvability, giving rise to an open-ended evolutionary path $[38]^{\mathrm{a}}$. The key point is, however, that within the above mentioned constraints, the DKS selection rule from DK less stable to DK more stable - can also be seen to rest on a mathematical truism, the mathematics of exponential growth. The DKS selection rule thereby becomes an additional sub-set of the global selection rule, 'less stable to more stable', discussed earlier.

Given the different mathematical foundation for each of the two stability kinds, it should come as no surprise that the evolutionary process for each of the two material kinds follows different kinetic patterns. For an isolated system (exchanging neither matter nor energy with the environment) the system is directed toward the lowest energy state - the equilibrium state, where entropy is maximal, and the drift toward that state tends to be monotonic. For a persistent DK system, however, governed as it is by divergent and intrinsically non-linear autocatalytic processes, the drift toward its stationary state can result in periodic or even chaotic behavior [29,39-41]. And being divergent, the system is not directed toward one specific state, but, rather, any number of feasible evolutionary pathways are possible. Moreover, the DKS formulation suggests that, in contrast to an isolated thermodynamic system, where the maximal (energetic) stability of the equilibrium state is achievable, in biological systems maximal stability (in the time/persistent sense) is unachievable. There can be no formal stability maximum in DKS systems given the almost infinite possibilities of variability that the divergent and open system description offers and the nature of stability in its time/persistence facet.

To summarize, whereas thermodynamic stability (for isolated systems) involves a probabilistic reordering of the existing, a drive toward entropically measured randomness, and is defined in energy terms, DKS is governed by the kinetic power of exponential growth acting on particular replicative systems and is manifest through its 
persistence over time. It is that kinetic power which both establishes the DK state and then drives it so as to channel that kinetic power most effectively, i.e., to exploit energetic and material resources most efficiently. Thus the empirical observation of an evolutionary process toward enhanced stability within the replicative world (whether replicative chemical or biological) also has its roots in a mathematical truism. Indeed DKS may be thought of as a Malthusian stability, in recognition of the contribution of Malthus to the appreciation of the consequences of exponential growth on replicating populations [42], and its subsequent influence on Darwin's formulation of the concept of natural selection. The result - a continually expanding replicative network able to penetrate and exploit most any ecological niche, whether deep under the sea, high in the earth's atmosphere, in polar ice-caps or tropical forests, above ground or miles below the earth's surface - two mathematically-based stability kinds leading to two distinct material forms.

\section{Relationship between the two stability kinds}

We have attempted to explain why there are just two material categories in nature, as well as the basis of those two categories, so let us now apply that insight to address aspects of the relationship that links those two material kinds. The fact that there are two stability kinds, each underpinned by its particular mathematical logic, means that the corresponding material forms can be expected to exhibit very different characteristics. And indeed they do. Whereas the properties of non-living things are largely explicable in well-established physical and chemical terms, the world of living things has proven resistant to similar characterization. We return to the issue of teleonomy, the term popularised by Monod specifically to describe the behavior of biological systems [14]. All living systems appear to have an agenda, to be goal-directed, as evident in their actions - building a nest, raising young, fighting off predators, and so on. But how can living things, ultimately nothing more than a form of material organization, act in a goal-directed fashion? How does life's unequivocal teleonomic character cohabit with the essence of the modern scientific revolution - nature's objective character?

It turns out that cohabitation need not be contradictory, that nature can be both objective and goal-directed. Once it is recognized that change is written into nature's laws, and that nature is goal-directed in a way that is logically prescribed - toward systems of greater stability, then the existence of two worlds, one living, one nonliving, becomes explicable. There are two distinct kinds of stabilities in nature, so nature's goal directedness reflects that duality - in the non-living world nature follows the thermodynamic directive (termed by Mayr teleomatic [15]), the probabilistic drive toward uniformity, toward so-called heat death, whereas for persistent replicating systems, nature's drive is toward replicative stability (DKS), with its teleological undertones (though consistent with the requirements of the Second Law). Thus nature's goaldirectedness with respect to persistent replicating systems, though teleologically tinged, can now be understood as manifesting an aspect of its objective character - the fundamental drive of all material systems toward ever greater stability.

Once the issue of goal-directedness in the biological world is resolved, two of biology's seemingly incompatible bedfellows - stability and complexity - can be harmoniously wedded, and this can be brought about through the mediating concept of function. As one of us has recently described, in the replicating world stability and function are directly related - greater replicative stability is induced through enhanced replicative function [20]. But, as is readily verifiable, there is also a logical connection between function and complexity. Function, of whatever kind, biological or technological, is almost invariably enhanced through complexity. Indeed, to paraphrase Carl Sagan's famous aphorism, one could say: extraordinary function requires extraordinary complexity, thereby offering insight into the connection between life's extraordinary functionality and its staggering complexity. But from these two relationships it then follows that (replicative) stability and complexity are also linked - greater complexity is necessary for greater stability. The physical-biological relationality can be summed up by the triad: stability - function - complexity, all interconnected and interrelating [20].

As a final point, let us now address a purely material aspect, the issue of material transfer between the two worlds, as evidenced on this planet. First, why was inanimate transformed into animate matter in the first place, i.e., why did life emerge. Second, it is obvious that once life was established on our planet, there has been a continual transfer of matter between the two material forms; living things die and their material form reverts to inanimate, while in the reverse direction, inanimate matter is drawn into the web of life, and thereby transformed into animate matter. But which process is dominant, and why? What can one say about the rates of material transformation in the two directions starting from that moment when earliest life was able to emerge?

The fact that life presumably started off in some limited physical location and expanded rapidly to occupy just about every conceivable planetary niche capable of sustaining life states unambiguously that once a stable and evolvable DKS system emerged on earth the rate of inanimate to animate transformation exceeded the reverse process, i.e., the rate of animate degradation. The fact that this difference is fundamental, not merely incidental, is confirmed by a recent estimate of the ongoing rate of growth of the earth's biomass, ca. $10^{17} \mathrm{~g} C /$ year 
[43]. That rate of growth turns out to be a significant percentage of the earth's total estimated biomass, ca. $10^{18} \mathrm{~g} \mathrm{C}$ [44]. In other words from the moment that life on earth emerged, there is every indication that for much of the time animate formation exceeded animate degradation. In fact, one might even see in man's attempt to physically explore the universe beyond our planet as an expression of animate matter's tendency to expand into all available niches, to continue life's relentless drive to expand wherever possible.

The reason for the clear imbalance in the rate of animate formation compared its decay, can now be pointed out. Based on the stability kinds involved, the conversion of animate to inanimate - death - is expected to be slower than the process of animate formation leading to life. Animate to inanimate is governed by the Second Law, by the more muted directive, the one concerned with material reorganization based on probabilistic considerations, while inanimate to animate is autocatalytic and driven by the kinetic power of exponential growth. Thus once a stable DK system emerges, i.e., once a network of far-from-equilibrium metabolic reactions that is holistically replicative is firmly established, the on-going drive toward greater DKS wins out, and the Second Law directive is circumvented and marginalized. In fact an energy-gathering metabolic capability must be an intrinsic component of the DKS system [12,13] for the Second Law requirement to be satisfied. Or put another way, once the necessary conditions for life's emergence are met and life is established, the kinetic drive toward more life, and more efficient life, overshadows the thermodynamic directive toward death, though of course that continuing transformation is conditional on a continuing source of energy. And the environmental consequences of that kinetic imbalance is dramatic and clear to see - life is (effectively) everywhere. The cosmological implications of these simple ideas need further consideration, but the preliminary conclusion seems to be that, provided a continual source of energy is available (most likely fed by nuclear processes in suns), matter will preferentially be driven from inanimate to animate, from non-replicative to replicative, that life will invariably prevail over non-life.

Notwithstanding the above comments, it should also be made clear that the dominance of animate formation over its degradation should not be seen as smoothly monotonic, but rather one that can itself be highly contingent, as is evident in the evolutionary process itself. It is generally believed that in the long evolutionary process toward ever more effective replicating networks there may have been periods of regression as a result of drastic ecological and/or climatic changes, such as the emergence of oxygen as a significant component of the earth's atmosphere [45]. Such an event could likely have led to the destruction of anaerobic life forms that populated the early planet. But the underlying long-term trend is unmistakable - the exponential driving force of living processes overwhelms the mathematically weaker Second Law directive.

\section{The question of life's contingency}

The above discussion on life's emergence, and its explosive (and continuing) expansion since its emergence, leads us to the problematic issue of life's contingency. In fact it is the issue of contingency which remains the central unresolved dilemma in our attempt to place animate systems squarely within a comprehensive material framework. In order to connect between the inanimate and animate worlds, it is presumed that the life process would have begun with the contingent emergence of a persistent and evolvable DK system, even though the likelihood of such a system emerging spontaneously currently remains unknown. So how contingent is life? What materials and reaction conditions would facilitate the emergence of a suitable DKS system? We do not know and we are still far from being able to answer these questions. That is the prime reason we are unable to specify how likely it is for life to exist elsewhere in the universe. But we may be at a turning point. Through recent advances in systems chemistry $[46,47]$, the path to enlightenment now seems more clearly marked, with preliminary results, both experimental [48] and theoretical [49], suggesting that replicative networks are under certain circumstances able to emerge spontaneously. Thus the immediate goal: the synthesis of stable DK systems so as to enhance our understanding of how DKS systems can be generated, and how readily they can be maintained. The DKS state is a chemically intricate and dynamic entity so its synthesis cannot be assumed to be a trivial one. Theory now needs to give way to experiment, very much in line with Richard Feynman's aphorism: "What I cannot create, I do not understand". And with regard that most intriguing of questions: how likely is it that life exists elsewhere in the universe, paradoxically, it could well be that through experiments conducted on earth, that we may finally reveal the likelihood of life existing elsewhere in the universe. In any case, a prevailing perception that protolife might be created through incorporating some replicating entity and its building blocks within a vesicle-like structure, seems unlikely to be productive, as several of the prerequisites of the DK state would be absent. The evolutionary process by which life was able to undergo such extraordinary complexification can only be understood in the context of exponential replicating systems.

\section{Conclusion}

This perspective has attempted to demonstrate that through an appreciation of nature's axiomatic drive toward stable/persistent forms, the underlying connection 
between the two organizational forms of matter - animate and inanimate - can be understood. In simplest terms, nature is able to express its spontaneous drive toward ever increasing stability, not in one, but in two fundamentally different ways, one based on energetic considerations - thermodynamic stability; the other based on time/persistence considerations - dynamic kinetic stability (DKS), with each leading to a particular manifestation of material organization. That basic reality means that material organization and reactivity take place in two seemingly parallel, yet intersecting, worlds. One hundred years after Ludwig Boltzmann laid down the statistical basis for the Second Law, and two hundred years after Thomas Malthus pointed out the profound consequences of exponential growth on living populations, it is now possible to see that within those two fundamental mathematical truths can be found not just the basis for a dual material world, but also the basis for change both within, and between, those two worlds. Life, in its stupendous diversity and extraordinary complexity, is just the inevitable consequence of mathematical law (exponential growth) operating on very particular replicating chemical systems. The answer to Schrödinger's 'what is life' question may finally be within reach.

\section{Endnotes}

${ }^{a}$ The ways in which DKS behavior and evolvability could emerge in a far-from-equilibrium system are certainly diverse and the possibility that both of them can appear at the same time cannot be excluded. See ref. [50].

\section{Competing interests}

The authors declare they have no competing interests.

\section{Authors' contributions}

This paper is the result of extensive discussions and correspondence over time between the two authors. AP wrote an initial draft and RP modified and extended the draft. The final paper reflects the views of both authors. Both authors read and approved the final manuscript.

\section{Acknowledgements}

The authors thank Prof. Jan Engberts for helpful comments on an early draft and the COST Action CM0703 "Systems chemistry" for providing a fruitful context for scientific exchanges during the realization of this work.

\section{Author details}

'Institut des Biomolécules Max Mousseron, UMR5247 CNRS-Universités Montpellier 1 \& Montpellier 2, CC17006, Place E. BataillonCC17006, Place E. Bataillon, Montpellier F-34095, France. ${ }^{2}$ Department of Chemistry, Ben-Gurion University of the Negev, Be'er Sheva 84105, Israel.

Received: 30 November 2013 Accepted: 12 March 2014 Published: 28 March 2014

\section{References}

1. Darwin C: On the origin of species. Cambridge, MA: Harvard University Press; 1859.

2. Kauffman SA: Investigations. Oxford: Oxford University Press; 2000

3. Ruiz-Mirazo K, Briones C, de la Escosura A: Prebiotic Systems Chemistry: New Perspectives for the Origins of Life. Chem Rev 2014, 114(1):285-366. doi:10.1021/cr2004844.
4. Pross A, Pascal R: The origin of life: what we know, what we can know, what we will never know. Open Biol 2013, 3:120190. doi:10.1098/ rsob.120190

5. Pross A: What is life? How chemistry becomes biology. Oxford: Oxford University Press; 2012.

6. Forterre P, Gribaldo S: The origin of modern terrestrial life. HFSP J 2007, 1:156-168. doi:10.2976/1.2759103.

7. Shapiro R: Small molecule interactions were central to the origin of life. Q Rev Biol 2006, 81:105-125. doi:10.1086/506024.

8. Luisi PL: The emergence of life: from chemical origins to synthetic biology. Cambridge, UK: Cambridge University Press; 2006.

9. Popa R: Between necessity and probability: searching for the definition and origin of life. Berlin: Springer; 2004

10. Fry I: The emergence of life on Earth. Piscataway, NJ: Rutgers University Press; 2000.

11. Schrödinger E: What is life? The physical aspects of the living cell. Cambridge, UK: Cambridge University Press; 1944.

12. Pascal R: Suitable energetic conditions for dynamic chemical complexity and the living state. J Syst Chem 2012, 3:3. doi:10.1186/1759-2208-3-3.

13. Pascal R, Pross A, Sutherland JD: Towards an evolutionary theory of the origin of life based on kinetics and thermodynamics. Open Biol 2013, 3:130156. http://dx.doi.org/10.1098/rsob.130156.

14. Monod J: Chance and necessity. New York: Random; 1972.

15. Mayr E: Toward a new philosophy of biology. Cambridge: Harvard University Press; 1988.

16. Pross A: Toward a general theory of evolution: Extending Darwinian theory to inanimate matter. J Syst Chem 2011, 2:1.

17. Pross A: Seeking the Chemical Roots of Darwinism: Bridging between Chemistry and Biology. Chem Eur J 2009, 15:8374-8381.

18. Pross A: Stability in chemistry and biology: Life as a kinetic state of matter. Pure Appl Chem 2005, 77:905-1921.

19. Pross A, Khodorkovsky V: Extending the concept of kinetic stability: toward a paradigm for life. J Phys Org Chem 2004, 17:312-316.

20. Pross A: The evolutionary origin of biological function and complexity. J Mol Evol 2013, 76:185-191. doi:10.1007/s00239-013-9556-1.

21. Wagner N, Pross A, Tannenbaum E: Selective advantage of metabolic over non-metabolic replicators: a kinetic analysis. Biosystems 2009, 99:126-129.

22. Dawkins R: The selfish gene. Oxford: Oxford University Press; 1989.

23. Sethna J: Statistical mechanics. Oxford: Oxford University Press; 2006:78.

24. Bissette AJ, Fletcher SP: Mechanisms of Autocatalysis. Angew Chem Int Ed 2013, 52:12800-12826. doi:10.1002/anie.201303822.

25. Plasson R, Brandenburg A, Jullien L, Bersini H: Autocatalysis: At the root of self-replication. Artificial life 2011, 17:219-236.

26. Eigen M: Selforganisation of matter and the evolution of biological macromolecules. Naturwissenschaften 1971, 58:465-523.

27. Kauffman SA: Cellular homeostasis, epigenesis and replication in randomly aggregated macromolecular systems. J Cybernetics 1971 , 1:71-96.

28. Gánti T: The principles of life. Oxford: Oxford University Press; 2003.

29. Lotka AJ: Contribution to the theory of periodic reaction. J Phys Chem 1910, 14:271-4

30. Eigen $M$, Schuster P: The hypercycle. A principle of natural selforganization. Part A: Emergence of the Hypercycle. Naturwissenschaften 1977, 64:541-565. doi:10.1007/BF00450633.

31. von Kiedrowski G: A self-replicating hexadeoxynucleotide. Angew Chem Int Ed 1986, 25:932-935.

32. Szathmáry E, Gladkih I: Sub-exponential growth and coexistence of non-enzymatically replicating Templates. J Theor Biol 1989, 138:55-58.

33. Lifson S: On the crucial stages in the origin of animate matter. J Mol Evol 1997, 44:1-8.

34. Vasas V, Szathmáry E, Santos M: Lack of evolvability in self-sustaining autocatalytic networks: A constraint on the metabolism-first path to the origin of life. Proc Natl Acad Sci USA 2010, 107:1470-1475.

35. Vasas V, Fernando C, Santos M, Kauffman S, Szathmáry E: Evolution before genes. Biol Direct 2012, 7:1. doi:10.1186/1745-6150-7-1.

36. Breslow R: On the mechanism of the formose reaction. Tetrahedron Lett 1959, 1(21):22-26.

37. Schrum JP, Zhu TF, Szostak JW: The Origins of Cellular Life. Cold Spring Harb Perspect Biol 2010, 2:a002212. doi:10.1101/cshperspect.a002212.

38. Mavelli F, Ruiz-Mirazo K: Theoretical conditions for the stationary reproduction of model protocells. Integr Biol 2013, 5:324-341. 
39. Goldbeter A: Biochemical oscillations and cellular rhythms. Cambridge, UK: Cambridge University Press; 1996.

40. Tyson Jj: In Biochemical oscillations: in Computational cell biology. Edited by Fall CP, Marland ES, Wagner JM, Tyson JJ. New York: Springer-Verlag; 2002.

41. McKane AJ, Nagy JD, Newman TJ, Stefanini MO: Amplified biochemical oscillations in cellular systems. J Stat Phys 2007, 128:165-191.

42. Malthus T: An essay on the principle of population. London: Printed for Johnson J, in St. Paul's Church-Yard; 1798. http://www.esp.org/books/ malthus/population/malthus.pdf.

43. Field CB, Behrenfeld MJ, Randerson JT, Falkowski P: Primary production of the biosphere: Integrating terrestrial and oceanic components. Science 1998, 281:237-240. doi: 10.1126/science.281.5374.237.

44. Kallmeyer J, Pockalny R, Adhikari RR, Smith DC, D'Hondt S: Global distribution of microbial abundance and biomass in subseafloor sediment. Proc Natl Acad Sci USA 2012, 109:162131-16216. doi: 10.1073/ pnas.1203849109.

45. Sessions AL, Doughty DM, Welander PV, Summons RE, Newman DK: The continuing puzzle of the great oxidation event. Current Biol 2009, 19:R567-R574.

46. Stankiewicz J, Eckardt LH: Chembiogenesis 2005 and systems chemistry workshop. Angew Chem Int Ed 2006, 45:342.

47. Ludlow RF, Otto S: Systems chemistry. Chem Soc Rev 2008, 37:101-108.

48. Vaidya N, Manapat ML, Chen IA, Xulvi-Brunet R, Hayden EJ, Lehman N: Spontaneous network formation among cooperative RNA replicators. Nature 2012, 491:72-77.

49. Hordijk W, Steel M: Detecting autocatalytic, self-sustaining sets in chemical reaction systems. J Theor Biol 2004, 227(4):451-461.

50. Wu M, Higgs PG: Origin of Self-Replicating Biopolymers: autocatalytic Feedback Can Jump-Start the RNA World. J Mol Evol 2009, 69:541-554.

doi:10.1186/1759-2208-5-3

Cite this article as: Pascal and Pross: The nature and mathematical basis for material stability in the chemical and biological worlds. Journal of Systems Chemistry 2014 5:3.

\section{Publish with ChemistryCentral and every scientist can read your work free of charge \\ "Open access provides opportunities to our colleagues in other parts of the globe, by allowing anyone to view the content free of charge." \\ W. Jeffery Hurst, The Hershey Company. \\ - available free of charge to the entire scientific community \\ - peer reviewed and published immediately upon acceptance \\ - cited in PubMed and archived on PubMed Central \\ - yours - you keep the copyright \\ Submit your manuscript here: \\ http://www.chemistrycentral.com/manuscript/<smiles>c1ccccc1</smiles> \\ Chemistry Central}

